\title{
Strong epistatic and additive effects of linked candidate SNPs for Drosophila pigmentation have implications for analysis of genome-wide association studies results
}

\author{
Jean-Michel Gibert ${ }^{2 \dagger}$, Jorge Blanco ${ }^{1 \dagger}$, Marlies Dolezal ${ }^{1}$, Viola Nolte ${ }^{1}$, Frédérique Peronnet ${ }^{2}$ \\ and Christian Schlötterer ${ }^{1 *}$ (D)
}

\begin{abstract}
Background: The mapping resolution of genome-wide association studies (GWAS) is limited by historic recombination events and effects are often assigned to haplotype blocks rather than individual SNPs. It is not clear how many of the SNPs in the block, and which ones, are causative. Drosophila pigmentation is a powerful model to dissect the genetic basis of intra-specific and inter-specific phenotypic variation. Three tightly linked SNPs in the t-MSE enhancer have been identified in three $D$. melanogaster populations as major contributors to female abdominal pigmentation. This enhancer controls the expression of the pigmentation gene tan $(t)$ in the abdominal epidermis. Two of the three SNPs were confirmed in an independent study using the D. melanogaster Genetic Reference Panel established from a North American population.
\end{abstract}

Results: We determined the functional impact of SNP1, SNP2, and SNP3 using transgenic lines to test all possible haplotypes in vivo. We show that all three candidate SNPs contribute to female Drosophila abdominal pigmentation. Interestingly, only two SNPs agree with the effect predicted by GWAS; the third one goes in the opposite direction because of linkage disequilibrium between multiple functional SNPs. Our experimental design uncovered strong additive effects for the three SNPs, but we also found significant epistatic effects explaining up to $11 \%$ of the total variation.

Conclusions: Our results suggest that linked causal variants are important for the interpretation of GWAS and functional validation is needed to understand the genetic architecture of traits.

Keywords: Genome-wide associations studies (GWAS), Genetic architecture, Additivity, Epistasis, Pigmentation, Drosophila

\section{Background}

Over the past years, genome-wide association studies (GWAS) have become the method of choice to identify functionally relevant variation for a wide set of traits [1, 2]. Despite the increased resolution of GWAS, compared with experimental crosses, the mapping resolution is frequently not sufficiently high to map the causative variants. The haplotype blocks

\footnotetext{
* Correspondence: Christian.Schloetterer@vetmeduni.ac.at

${ }^{\dagger}$ Equal contributors

'Institut für Populationsgenetik, Vetmeduni Vienna, Veterinärplatz 1, 1210

Wien, Austria

Full list of author information is available at the end of the article
}

identified by GWAS often carry multiple variants and it is not clear how many of them and which ones are causative. Many approaches have been proposed to prioritize candidate single nucleotide polymorphisms (SNPs). While rather advanced approaches are available for protein-coding variants (e.g. SIFT, PolyPhen2, MutationAssessor [3-5]), the characterization of regulatory SNPs suffers from low sequence conservation and incomplete knowledge about functionally important motives. The large number of candidate SNPs also precludes experimental inference of epistasis since the number of possible haplotypes scales 
exponentially with the number of loci. Indeed, only a few studies analyzed the fitness consequences of all combinations of small sets of mutations (reviewed in [6]). Consequently, the experimental validation of GWAS results is often confined to cell culture or knockdown of candidate genes.

Drosophila pigmentation has a long tradition as model to study the genetic basis of phenotypic variation within and between species [7]. Pool-GWAS in three natural $D$. melanogaster populations identified three closely linked SNPs as major contributors to female abdominal pigmentation $[8,9]$. These three SNPs are located within a 208-bp window of the $t$-MSE enhancer, which controls the expression of the pigmentation gene $\tan (t)$ in the abdominal epidermis [10]: X-9121129 (SNP1), X9121094 (SNP2), and X-9120922 (SNP3) (Additional files 1 and 2: Table S1 and Figure S1). An independent study, which does not rely on Pool-Seq [11] of extreme phenotypes but analyzed individual isofemale lines, confirmed the importance of this regulatory region for natural variation in female abdominal pigmentation [12]. Using the D. melanogaster Genetic Reference Panel, which was established from a North American population, two of the SNPs identified by Pool-GWAS (SNP1, SNP2) were also detected [12].

Building on the previously identified GWAS signal in the regulatory region of the pigmentation gene $\tan (t)$, we performed the first in vivo characterization of all GWAS candidate SNPs in a quantitative trait locus (QTL) region. It could have been expected that a single causative variant was linked to two neutral SNPs. However, we find that all three candidate SNPs have strong additive and complex epistatic effects on pigmentation. Interestingly, only two agree with the effect predicted by GWAS; the third one goes in the opposite direction.

\section{Results}

We used transgenic lines to evaluate the functional impact of SNP1, SNP2, and SNP3. We constructed rescue transgenes, each containing the $t-M S E$ with one of the eight combinations of the three SNPs fused to the $h s p 70$ minimal promoter and the $t$ complementary DNA (cDNA). Each transgene was inserted at the same genomic position using phiC31 integrase-based transgenesis [13]. The effect of the different SNP combinations on abdominal pigmentation was assessed in females with an otherwise identical genetic background, mutant for $t$ (allele $\left.t^{d 07784}[14]\right)$. The different genotypes showed pronounced pigmentation differences (Fig. 1, left). For a quantitative analysis of pigmentation differences, we analyzed 15 individuals for each genotype and quantified the pigmentation in segments A5, A6, and A7 on mounted abdominal segments (Additional file 3: Raw Data; Fig. 1, right). We detected clear differences between the genotypes, covering a similar phenotypic space as wild-type (WT) flies (Fig. 1). We determined the main effects of all SNPs and their interactions using ANOVA (Additional file 4: Table S2). All SNPs had very significant main effects in all segments $(p<0.001)$, explaining up to $71 \%$ of the total variance in pigmentation (Table 1). SNP1 had the strongest main effect, explaining $29-53 \%$ of the variation. Epistatic effects were considerably weaker accounting for $6-11 \%$ of the total variation. For all segments, a model with epistatic effects provided a significantly better fit to the data than a model with main effects only $(p \leq 0.001)$. The presence of epistatic effects in a regulatory element is in line with results for transcription factor binding sites [15].

The effects of the three SNPs on the pigmentation were largely consistent across segments. Nevertheless, some differences between segments were noticed (Fig. 1, Table 1, Additional file 4: Table S2). SNP1 had the strongest main effect in A6 and the weakest main effect in A7, whereas the opposite was observed for SNP3. SNP2 had the strongest main effect in A7 and the weakest main effect in A5. The two-way interactions involving SNP1 were only significant in A6, while all other interactions were significant across all three segments (Table 1). We attribute this heterogeneity among segments to differences in expression of genes regulating tan. The transcription factors Abdominal-B and bric-à-brac (BAB1 and $B A B 2$ ), which regulate abdominal pigmentation and are expressed at an increasing level along the anteroposterior axis in pupal epidermis [16, 17], are good candidates, although the polymorphic SNPs are not located in characterized binding sites.

Importantly, not all Dark alleles predicted by GWAS increased pigmentation in our functional assays. While the Dark alleles of SNP1 and SNP3 increase pigmentation, the Dark allele of SNP2 has the opposite effect. Indeed, the darkest genotype corresponds to the combination D.L.D, whereas the lightest genotype corresponds to the opposite combination L.D.L. Thus, opposite to the predictions of three GWAS studies, SNP2 does not affect pigmentation in the same direction as the linked SNPs. A closer inspection of the underlying haplotype structure shows that despite the high recombination rate and large population size of $D$. melanogaster, the absence of the haplotypes with extreme pigmentation prevented the correct inference of the effects of the three causative SNPs [9].

\section{Discussion}

The functional impact of natural variants on a given trait is one of the most pressing questions in genetics. GWAS and QTL mapping studies substantially advanced our understanding of the contribution of genes at different, well-separated locations in the genome. Nevertheless, 


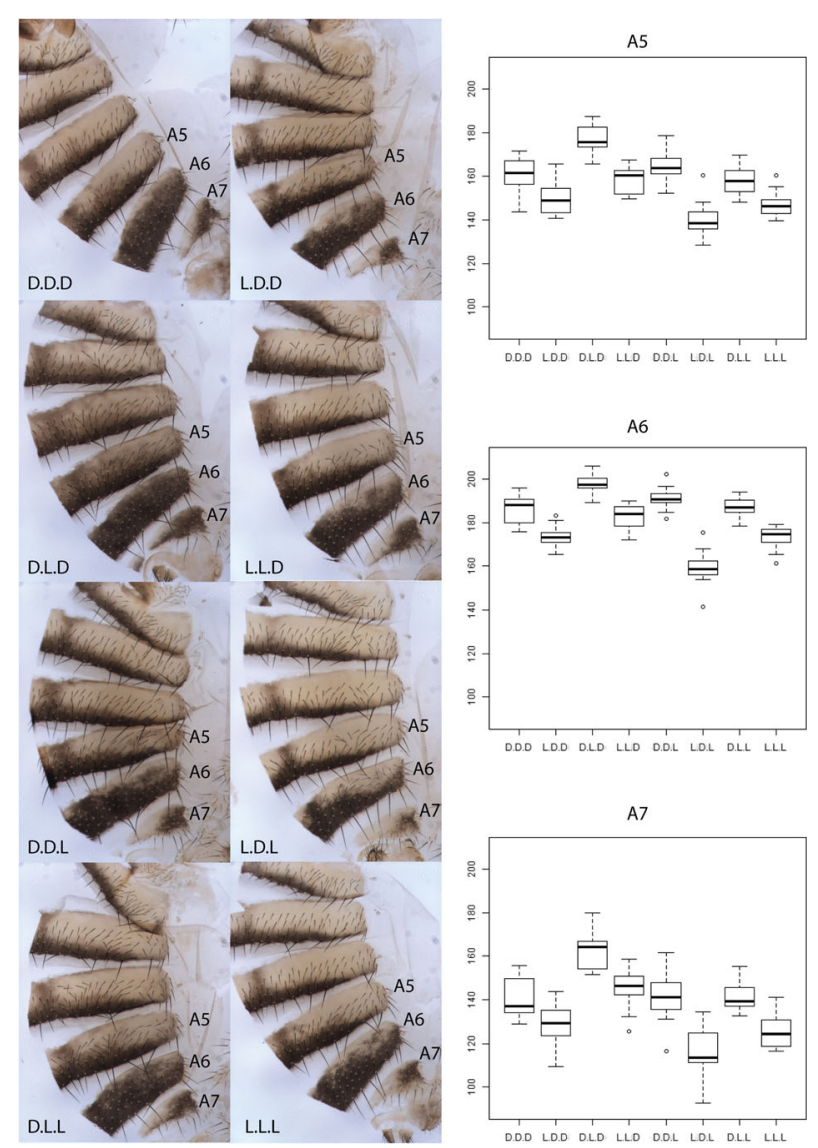

Fig. 1 Effect of the three SNPs on abdominal pigmentation. Left: abdominal cuticles of the different genotypes carrying the eight possible combinations of the three tested SNPS $(1,2$, and 3). A5, A6, and A7: abdominal segments 5, 6, and 7. Right: box plots of pigmentation of A5, A6, and A7 for the eight genotypes carrying all possible combinations of the three tested SNPs. The letters D and L correspond to the prediction of the GWAS studies (Dark or Light)

the analysis of linked variants has been severely limited by linkage disequilibrium. In this report, we use, to our knowledge for the first time, transgenesis to break up linkage disequilibrium among closely linked sites to determine their contribution to the trait of interest (pigmentation) and to study the underlying genetic architecture. Our result of all candidate SNPs of the tan locus contributing to the pigmentation demonstrates that multiple SNPs could be contributing to a single QTL. The current literature already indicates that this phenomenon may be not uncommon: several multiparent advanced intercrosses noted that a given QTL explained a significant fraction of the variation, but variants segregating in the corresponding region did not [18-21]. The authors interpreted this discrepancy as evidence for multiple functional alleles contributing to the trait [18-20]. Similarly, multiple alleles have been suggested to contribute to a given

Table 1 Summary of the three-way ANOVAs (full factorial model) performed independently on segments A5, A6, and A7

\begin{tabular}{|c|c|c|c|c|c|c|}
\hline & \multicolumn{2}{|l|}{ A5 } & \multicolumn{2}{|l|}{ A6 } & \multicolumn{2}{|l|}{ A7 } \\
\hline & $p$ value & Eta Squared & $p$ value & Eta Squared & $p$ value & Eta Squared \\
\hline 1 & $<0.001$ & 0.422 & $<0.001$ & 0.533 & $<0.001$ & 0.295 \\
\hline 2 & $<0.001$ & 0.059 & $<0.001$ & 0.097 & $<0.001$ & 0.132 \\
\hline 3 & $<0.001$ & 0.130 & $<0.001$ & 0.081 & $<0.001$ & 0.180 \\
\hline $1 \times 2$ & 0.206 & 0.004 & $<0.001$ & 0.025 & 0.394 & 0.002 \\
\hline $1 \times 3$ & 0.260 & 0.003 & $<0.001$ & 0.032 & 0.074 & 0.009 \\
\hline $2 \times 3$ & $<0.001$ & 0.058 & 0.013 & 0.010 & $<0.001$ & 0.047 \\
\hline $1 \times 2 \times 3$ & $<0.001$ & 0.044 & $<0.001$ & 0.042 & 0.025 & 0.015 \\
\hline
\end{tabular}

1, 2, and 3 in the first column correspond to SNP1, SNP2, and SNP3. $P$ values for the different effects are indicated as well as Eta Squared, a measure of the effect size 
eQTL $[22,23]$. The strongest independent support for multiple functional variants comes from a study on the Alcohol dehydrogenase $(A d h)$ in D. melanogaster, where the authors divided the gene into three parts with multiple SNPs and showed that each part contributed to Adh expression [24].

Analyzing all eight possible combinations of the three adjacent functional intra-specific variants provided the first in vivo analysis of the genetic architecture of a regulatory module in a higher eukaryote. Although additive effects explain most of the trait variation, up to $11 \%$ of the variation arises from epistatic interactions between the three sites. The regulatory architecture of pigmentation is similar in the three abdominal segments, but some noticeable differences are present. The about tenfold difference in the variance, explained by epistatistic two-way interactions involving SNP1, results in a significant effect only in A6. In addition to cuticle pigmentation, tan is also involved in vision [14]. The expression of $\tan$ in photoreceptors is responsible for the hydrolysis of carcinine, a different substrate than Beta-alanyldopamine which is responsible for cuticle pigmentation $[25,26]$. We anticipate that such other roles of tan could have their own regulatory architecture, which differs from the one of abdominal pigmentation.

Our observations have direct implications for other GWAS studies: (1) consistent with a previous report concluding that complex traits are mainly governed by additive effects [27], we find that the main effects are much stronger than epistasis; (2) linkage disequilibrium between causal SNPs could result in predicting effects in the wrong direction. Very importantly, this is not an artifact limited to this specific case in Drosophila, it will apply to all GWAS studies [28]. The confounding effect of multiple causative alleles at the same locus has also been described for several other systems (e.g. $[28,29])$.

\section{Conclusion}

We propose that the possibility of linked causal variation needs to receive more attention and emphasize the importance of experimental systems, such as fruit flies, which allow functional testing of candidate SNPs in vivo as well as the inference of the underlying genetic architecture.

\section{Methods}

\section{Determination of the ancestral state}

In order to determine the ancestral state of the SNPs, the $t_{-} M S E$ of $D$. melanogaster was aligned with those of D. simulans, D. sechellia, and D. yakuba using clustalw (http://www.genome.jp/tools/clustalw/). These sequences were obtained by blasting the $t_{-} M S E$ of $D$. melanogaster against the genomes of these species (https://blast.ncbi.nlm.nih.gov/Blast.cgi).

\section{Drosophila strains and culture}

Flies were reared on standard medium at $25{ }^{\circ} \mathrm{C} . y^{1} w^{1118}$ flies were used as a source of genomic DNA. Targeted transgenesis was carried out using the line $\mathrm{ZH}$-attP$86 F b$ (BL-24749), which harbors the attP landing site at the cytological location $86 \mathrm{~F} 8$. The tan mutant allele $t^{d 07784}$ was obtained from the Bloomington Drosophila Stock center (BL-19282).

\section{Reporter constructs and targeted transgenesis}

The MSE element was amplified by PCR using genomic DNA from $y^{1} w^{1118}$ flies (with primers MSEO-F and $M S E O-R$ listed below) and cloned into the pGEMT-Easy vector (Promega). The resulting plasmid, $p G E M T-M S E$, was used as a template to generate MSE elements containing the different SNPs combinations following the site-directed plasmid mutagenesis technique (QuickChange $^{\mathrm{rx}}$, [30]). The MSE element from the $y^{1} w^{1118}$ flies carries the nucleotides predicted to give a dark pigmentation for each of the three SNPs [8]. The primer pairs MSE1-F and MSE1R, MSE2-F and MSE2-R, and MSE3-F and MSE3-R (listed below) were used to mutate them into the nucleotides predicted to give a light pigmentation. A reporter construct, generated using restriction-enzyme-based strategies, was used to analyze MSE enhancer activity. This vector derives from the site-specific transformation vector pattB (kindly provided by Johannes Bischof) and carries the $\tan$ cDNA (RH41996, DGRC\# 17763) as reporter gene. The different MSE elements were cloned upstream of the Hsp-70 minimal promoter and the tan cDNA. The resulting constructs were inserted at the same genomic location on the third chromosome via site-specific integration [13]. Such mini-genes where an enhancer is cloned just upstream the cDNA of the gene it drives have been previously fruitfully used as sensitive and quantitative readout of enhancer function [31,32].

\section{Establishment of the stocks for the phenotyping}

We established homozygous transgenic stocks by crossing the transgenic flies with balancer stocks. In order to homogenize the genetic background, the transgenic flies were backcrossed for ten generations to the stock $\mathrm{ZH}$ attP-86Fb (BL-24749), which had been used for injections. The transgenes were followed by the red eye color from the functional $w$ locus of the vector. The $\mathrm{X}$ chromosome carrying a transgene with the phiC31 integrase was later replaced with an X-chromosome carrying the tan mutant allele ( $w^{1118} t^{d 07784}$ [14]), whereas the autosomes were maintained using balancer chromosomes. We checked by Sanger sequencing that the lines carried 
the right transgenes. We observed that flies mutant for tan and homozygous for the rescue transgenes had a very pronounced pigmentation, much darker than WT at $25{ }^{\circ} \mathrm{C}$. We therefore phenotyped flies heterozygous for the transgene and homozygous for the $t^{d 07784}$ allele. These flies were obtained by crossing transgenic flies homozygous for the transgenes and for the $t^{d 07784}$ allele with the $t^{d 07784}$ stock.

\section{Abdominal cuticle preparations}

Four-day-old flies were stored for eight days in 75\% ethanol. Abdomens were cut next to the dorsal midline and the abdominal cuticles were cleaned, dehydrated in $100 \%$ ethanol, and mounted in Euparal (Roth). Fifteen female flies were processed for each genotype condition.

\section{Imaging of fly abdomens}

Cuticle preparations were imaged with a binocular equipped with Leica DC480 digital camera using the Leica IM50 Image Manager software. An annular lamp was used to ensure homogeneous lighting. All pictures were taken in the same session and identical settings were used throughout the session. Pigmentation was quantified in A5, A6, and A7 hemisegments as the mean intensity using ImageJ software as previously reported [33]. For this, the hemisegments were delimited by hand and the function "measure" of ImageJ used. The obtained values were subtracted from 255 to get values comprised between 0 (white) and 255 (black).

\section{Statistical analyses}

All statistical analyses were performed with $\mathrm{R}$ 3.2.3 $[34,35]$. A full factorial three-way ANOVA was performed with sum zero constraints (option contr.sum) and estimation of Type III SS with package "car." Effect sizes were estimated as Eta Squared values, calculated as $\mathrm{SS}_{\text {effect }} / \mathrm{SS}_{\text {Total }}$.

\section{List of primers used in the study}

MSEO-F 5' GCGTTCCAACACCCCGTCTAATCTA 3' MSEO-R 5' CACGATTTCCGTATTTGAAATAATA 3' MSE1-F 5' ATAATATATTTATATTCTGATTATT 3' $M S E 1-R 5^{\prime}$ AATAATCAGAATATAAATATATTAT 3' MSE2-F 5' AATTATCCTAAGCCTTGATTCTATC 3' $M S E 2-R 5^{\prime}$ GATAGAATCAAGGCTTAGGATAATT 3' MSE3-F $5^{\prime}$ TCTAATTAGTATACATATTATGATC 3' MSE3-R 5' GATCATAATATGTATACTAATTAGA 3'

\section{Additional files}

Additional file 1: Table S1. Information related to the three most significant SNPs involved in female abdominal pigmentation variation in segment A7 identified previously [8]. (DOCX $50 \mathrm{~kb}$ )
Additional file 2: Figure S1 Alignment of $t$ MSE from D. melanogaster, D. simulans, D. sechellia, and D. yakuba. (DOCX $108 \mathrm{~kb})$

Additional file 3: Pigmentation measures in segments A5, A6, and A7 for the eight genotypes analyzed in this study. 1 and 2 in the SNP columns correspond to D or L alleles, respectively. (XLSX $43 \mathrm{~kb}$ )

Additional file 4: Table S2. ANOVAs performed independently on segments $A 5, A 6$, and $A 7$ to test the effect of the three SNPs on pigmentation. (DOCX $101 \mathrm{~kb})$

\section{Acknowledgments}

We are thankful to L. Endler for sharing results and members of the Institute of Population Genetics for feedback.

\section{Funding}

This work has been supported by the Austrian Science Funds (P-22725) and the European Research Council (ArchAdapt). The funding bodies had no role in the design of the study and collection, analysis, interpretation of data, and in writing the article.

Availability of data and materials

All analyzed data in this study are included in this published article (Additional file 3: Raw Data).

Authors' contributions

JB and JMG performed experiments. VN checked the lines by Sanger sequencing. JMG, MD, and CS analyzed the data. JMG and CS wrote the manuscript with the help of JB, VN, FP, and MD. All authors read and approved the final manuscript.

Ethics approval and consent to participate Not applicable.

Competing interests

The authors declare they have no competing interests.

\section{Publisher's Note}

Springer Nature remains neutral with regard to jurisdictional claims in published maps and institutional affiliations.

\section{Author details}

${ }^{1}$ Institut für Populationsgenetik, Vetmeduni Vienna, Veterinärplatz 1, 1210 Wien, Austria. ${ }^{2}$ Sorbonne Universités, UPMC Univ Paris 06, CNRS, Biologie du Développement Paris Seine-Institut de Biologie Paris Seine (LBD-IBPS), case 24, 9 quai St-Bernard, 75005 Paris, France.

Received: 20 March 2017 Accepted: 19 June 2017 Published online: 03 July 2017

References

1. Kruglyak $L$. The road to genome-wide association studies. Nat Rev Genet. 2008;9:314-8.

2. Visscher PM, Brown MA, McCarthy MI, Yang J. Five years of GWAS discovery. Am J Hum Genet. 2012;90:7-24.

3. Kumar P, Henikoff S, Ng PC. Predicting the effects of coding nonsynonymous variants on protein function using the SIFT algorithm. Nat Protoc. 2009:4:1073-81.

4. Adzhubei IA, Schmidt S, Peshkin L, Ramensky VE, Gerasimova A, Bork P, et al. A method and server for predicting damaging missense mutations. Nat Methods. 2010;7:248-9.

5. Reva B, Antipin $Y$, Sander $C$. Predicting the functional impact of protein mutations: application to cancer genomics. Nucleic Acids Res. 2011;39:e118.

6. de Visser JAGM, Krug J. Empirical fitness landscapes and the predictability of evolution. Nat Rev Genet. 2014:15:480-90.

7. Massey JH, Wittkopp PJ. The genetic basis of pigmentation differences within and between Drosophila species. Curr Top Dev Biol. 2016;119:27-61.

8. Bastide H, Betancourt A, Nolte V, Tobler R, Stobe P, Futschik A, et al. A genome-wide, fine-scale map of natural pigmentation variation in Drosophila melanogaster. PLoS Genet. 2013;9:e1003534. 
9. Endler L, Betancourt AJ, Nolte V, Schlötterer C. Reconciling differences in Pool-GWAS between populations: a case study of female abdominal pigmentation in Drosophila melanogaster. Genetics. 2016;202:843-55.

10. Jeong S, Rebeiz M, Andolfatto P, Werner T, True J, Carroll SB. The evolution of gene regulation underlies a morphological difference between two Drosophila sister species. Cell. 2008:132:783-93.

11. Schlötterer C, Tobler R, Kofler R, Nolte V. Sequencing pools of individuals mining genome-wide polymorphism data without big funding. Nat Rev Genet. 2014;15:749-63.

12. Dembeck LM, Huang W, Magwire MM, Lawrence F, Lyman RF, Mackay TFC. Genetic architecture of abdominal pigmentation in Drosophila melanogaster. PLoS Genet. 2015;11:e1005163.

13. Bischof J, Maeda RK, Hediger M, Karch F, Basler K. An optimized transgenesis system for Drosophila using germ-line-specific phiC31 integrases. Proc Natl Acad Sci U S A. 2007;104:3312-7

14. True JR, Yeh SD, Hovemann BT, Kemme T, Meinertzhagen IA, Edwards TN, et al. Drosophila tan encodes a novel hydrolase required in pigmentation and vision. PLoS Genet. 2005;1:e63.

15. Haldane A, Manhart M, Morozov AV. Biophysical fitness landscapes for transcription factor binding sites. PLoS Comput Biol. 2014;10:e1003683.

16. Williams TM, Selegue JE, Werner T, Gompel N, Kopp A, Carroll SB. The regulation and evolution of a genetic switch controlling sexually dimorphic traits in Drosophila. Cell. 2008;134:610-23.

17. Kopp A, Duncan I. Anteroposterior patterning in adult abdominal segments of Drosophila. Dev Biol. 2002;242:15-30.

18. King EG, Kislukhin G, Walters KN, Long AD. Using Drosophila melanogaster to identify chemotherapy toxicity genes. Genetics. 2014;198:31-43.

19. Giraud H, Lehermeier C, Bauer E, Falque M, Segura V, Bauland C, et al. Linkage disequilibrium with linkage analysis of multiline crosses reveals different multiallelic QTL for hybrid performance in the flint and dent heterotic groups of maize. Genetics. 2014;198:1717-34.

20. Rat Genome Sequencing and Mapping Consortium, Baud A, Hermsen R, Guryev V, Stridh P, Graham D, et al. Combined sequence-based and genetic mapping analysis of complex traits in outbred rats. Nat Genet. 2013;45:767-75.

21. Najarro MA, Hackett JL, Smith BR, Highfill CA, King EG, Long AD, et al. Identifying loci contributing to natural variation in xenobiotic resistance in Drosophila. PLoS Genet. 2015;11:e1005663.

22. Zeng B, Lloyd-Jones L, Holloway A, Marigorta UM, Metspalu A, Montgomery GW, et al. Constraints on eQTL fine mapping in the presence of multi-site local regulation of gene expression [Internet]. 2016. http://biorxiv.org/ lookup/doi/10.1101/084293

23. Wood AR, Esko T, Yang J, Vedantam S, Pers TH, Gustafsson S, et al. Defining the role of common variation in the genomic and biological architecture of adult human height. Nat Genet. 2014;46:1173-86.

24. Stam LF, Laurie CC. Molecular dissection of a major gene effect on a quantitative trait: the level of alcohol dehydrogenase expression in Drosophila melanogaster. Genetics. 1996;144:1559-64.

25. Wagner S, Heseding C, Szlachta K, True JR, Prinz H, Hovemann BT. Drosophila photoreceptors express cysteine peptidase tan. J Comp Neurol. 2007;500:601-11.

26. Aust S, Brüsselbach F, Pütz S, Hovemann BT. Alternative tasks of Drosophila tan in neurotransmitter recycling versus cuticle sclerotization disclosed by kinetic properties. J Biol Chem. 2010;285:20740-7.

27. Hill WG, Goddard ME, Visscher PM. Data and theory point to mainly additive genetic variance for complex traits. PLoS Genet. 2008;4:e1000008.

28. Lin P-I, Vance JM, Pericak-Vance MA, Martin ER. No gene is an island: the flip-flop phenomenon. Am J Hum Genet. 2007;80:531-8.

29. Kerdaffrec E, Filiault DL, Korte A, Sasaki E, Nizhynska V, Seren Ü, et al. Multiple alleles at a single locus control seed dormancy in Swedish Arabidopsis. elife. 2016;5(e22502).

30. Liu H, Naismith $\mathrm{JH}$. An efficient one-step site-directed deletion, insertion, single and multiple-site plasmid mutagenesis protocol. BMC Biotechnol. 2008;8:91.

31. Crocker J, Abe N, Rinaldi L, McGregor AP, Frankel N, Wang S, et al. Low affinity binding site clusters confer hox specificity and regulatory robustness. Cell. 2015;160:191-203.

32. Marcellini S, Simpson P. Two or four bristles: functional evolution of an enhancer of scute in Drosophilidae. PLoS Biol. 2006;4:e386.

33. Gibert J-M, Mouchel-Vielh E, De Castro S, Peronnet F. Phenotypic plasticity through transcriptional regulation of the evolutionary hotspot gene tan in Drosophila melanogaster. PLoS Genet. 2016;12:e1006218.
34. R Core Team. R: A language and environment for statistical computing. Vienna: R Foundation for Statistical Computing [Internet]; 2015. https:// www.R-project.org/.

35. Fox J, Weisberg S. An $\{R\}$ Companion to Applied Regression [Internet]. 2nd ed. Thousand Oaks, CA: Sage; 2011. http://socserv.socsci.mcmaster.ca/jfox/ Books/Companion.

\section{Submit your next manuscript to BioMed Central and we will help you at every step:}

- We accept pre-submission inquiries

- Our selector tool helps you to find the most relevant journal

- We provide round the clock customer support

- Convenient online submission

- Thorough peer review

- Inclusion in PubMed and all major indexing services

- Maximum visibility for your research

Submit your manuscript at www.biomedcentral.com/submit 